\title{
CME Dermatology (100625): self-assessment questionnaire
}

\author{
Edited by Christopher Griffiths and Tahseen A Chowdhury
}

\section{SAQs and answers are ONLINE for RCP fellows and collegiate members}

The SAQs printed in the CME section can only be answered online to achieve external CPD credits. Any comments should be sent in via email only: clinicalmedicine@rcplondon.ac.uk

\section{Format}

Candidates are asked to choose the best answer from the five possible answers. This best of five format is used in many medical examinations, however the questions are not intended to be representative of those used in the MRCP(UK) Part 1 or Part 2 Written Examinations.

\section{The answering process}

1 Go to www.rcplondon.ac.uk/SAQ

2 Log on using your usual RCP username and password

3 Select the relevant $\mathrm{CME}$ question paper

4 Answer all 10 questions by selecting the best answer from the options provided

5 Once you have answered all the questions, click on Submit

\section{Registering your external CPD credits}

Carrying out this activity allows you to claim two external CPD credits. These will be automatically transferred to your CPD diary, where you can review the activity and claim your points.

1 Which of the following statements is true regarding long-term sequelae of a case of Stevens-Johnson syndrome (SJS)?

(a) Asthma commonly develops in patients following an acute episode of SJS.

(b) Blindness when it occurs is due to retinal damage.

(c) Chronic lichen sclerosus is the primary cause of female sexual dysfunction in patients who have recovered from SJS.

(d) Patients may develop xerostomia following an episode of SJS.

(e) Peripheral neuropathy commonly develops secondary to thalidomide given in the acute phase of illness.
2 Which of the following statements is true of drug reaction with eosinophilia and systemic symptoms (DRESS)?

(a) Antibiotics are a rare cause of DRESS.

(b) Mucosal involvement is seen in $>80 \%$ of cases.

(c) The cutaneous eruption usually heals with scarring.

(d) The liver is the most common internal organ to be involved in the process.

(e) The mortality is $>30 \%$.

3 A 24-year-male patient has severe atopic eczema which appears to be unresponsive to topical steroids and emollients.

Which one of the following statements is correct?

(a) Biologic therapies are indicated in this case.

(b) It is important to identify the allergies responsible for this patient's poorly controlled eczema.

(c) Systemic immunosuppressive medication, including ciclosporin, methotrexate or azathioprine, should be considered.

(d) The patient should be admitted as an emergency and given antiviral medication to treat eczema herpeticum.

(e) Ultraviolet radiation (phototherapy) would not be appropriate.

4 In recent years research has contributed to significant advances in the understanding of eczema pathogenesis.

\section{Which of the following statements is true?}

(a) Genetically-determined skin barrier dysfunction increases risk for multiple allergic diseases.

(b) Genome-wide association studies have identified 15 loci which account for eczema risk.

(c) Intensive emollient use is an established method for the primary prevention of eczema.

(d) Mutations in the gene encoding filaggrin are responsible for causing asthma.

(e) Propionibacterium is the most common genus of bacteria associated with active eczema.

5 Which of the following factors is associated with a higher risk of hidradenitis suppurativa?
(a) childhood asthma
(b) high alcohol consumption
(c) previous history of deep vein thrombosis
(d) prolonged antibiotic therapy
(e) smoking. 
6 Which of the following is a first-line treatment for hidradenitis suppurativa?
(a) isotretinoin
(b) lymecycline
(c) omalizumab
(d) pulsed dye laser
(e) topical coal tar.

7 Which of the following mutations underlies $90 \%$ of basal cell carcinomas?
(a) BRAF
(b) MEK1
(c) p53
(d) PTCH1
(e) SMO.

8 Which of the following treatments affords the highest cure rate for basal cell carcinoma?
(a) cryotherapy
(b) Mohs micrographic surgery
(c) photodynamic therapy
(d) radiotherapy
(e) topical 5-fluorouracil.

9 A 28-year-old woman presented with a five-month history of progressive hair thinning prominently affecting the crown. Her periods were irregular and she had recently started treatment for severe acne. On examination there was diffuse hair loss over the vertex scalp, along with recession of her frontal hairline, worse at the temples. Close inspection revealed miniaturised hairs but no inflammation or scarring. Pull test was negative.

\section{Which of the following investigations should be considered?}
(a) connective tissue disease screen
(b) ferritin and zinc
(c) none
(d) pelvic ultrasound
(e) testosterone, sex hormone binding globulin and prolactin.

10 Janus kinase (JAK) inhibitors are a promising new treatment for which of the following alopecias?
(a) alopecia areata
(b) chronic cutaneous lupus erythematosus
(c) female pattern hair loss
(d) lichen planopilaris
(e) telogen effluvium.

\section{CME Renal medicine SAQ}

\section{Answers to the CME SAQ published in} Clinical Medicine October 2016

$\begin{array}{llllllllll}\text { Q1 } & \text { Q2 } & \text { Q3 } & \text { Q4 } & \text { Q5 } & \text { Q6 } & \text { Q7 } & \text { Q8 } & \text { Q9 } & \text { Q10 } \\ \text { (b) } & \text { (a) } & \text { (e) } & \text { (c) } & \text { (b) } & \text { (c) } & \text { (e) } & \text { (e) } & \text { (e) } & \text { (d) }\end{array}$

